\title{
Analysis of Comprehensive Optimization of Navigation Performance of High Speed Catamaran Unmanned Craft
}

\author{
Chengqi Cai, Shasha Gao, Songlin Yang, Yan Shi, Xiaoyu Huang
}

\begin{abstract}
The high-speed catamaran unmanned craft is taken as the research object. A comprehensive optimization mathematical model with rapidity, maneuverability and stability as the objective function was established. The genetic algorithm and particle swarm optimization algorithm were used to optimize the calculation respectively. Then, select the better ones and the algorithm was used to calculate the sensitivity of the five main variables (propeller diameter, pitch ratio, speed, speed, and body spacing) and the effects of different weight assignments on the objective function were discussed. The calculation results show that the particle swarm optimization algorithm is superior to the genetic algorithm. The propeller diameter is the most sensitive variable among the five variables. When the rapidity weight is $9 / 5$, the maneuverability is $4 / 5$, and the stability is $25 / 36$, the equation Constraint satisfaction is best.
\end{abstract}

Index Terms - high-speed catamaran unmanned craft; navigation performance; comprehensive optimization; optimization algorithm

\section{INTRODUCTION}

The unmanned craft is a complex system that integrates ship design, intelligent control and information processing. The ship design is a multidisciplinary and highly integrated science and technology, which includes research on seakeeping, rapidity, maneuverability and overturning. So its comprehensive navigation performance optimization design is also becoming more and more important ${ }^{[1]}$.For a long time, ship optimization design was based on model experiments and empirical formulas summarized by model experiments, and it has always been difficult to optimize its navigation performance through numerical prediction methods. Therefore, the introduction of multidisciplinary design optimization method has important research value ${ }^{[5]}$ for ship optimization design. Optimization algorithm is the core and foundation part of optimization design. With the continuous advancement of technology, the application of optimization algorithms in various engineering fields is increasingly widespread. At present, there are more and more researches on the optimization algorithm of catamaran. Zhang Weiyi uses the simulated annealing algorithm to design and optimize the structure of the catamaran ${ }^{[2]}$. Deng Fang studied the digital design of the catamaran based on the optimization of hull resistance performance ${ }^{[3]}$; Aiqin uses the PSO algorithm to comprehensively optimize the ship's resistance performance ${ }^{[4]}$ Most of the current research is mainly to study the structure and resistance performance of the catamaran unmanned craft,

Chengqi Cai, Shasha Gao, Songlin Yang, Yan Shi, Xiaoyu Huang School of Naval Architecture \& Ocean Engineering, Jiangsu University of Science and Technology, Zhenjiang, Jiangsu, China and the comprehensive performance of the catamaran unmanned craft is less. This paper compared the genetic algorithm with the particle swarm optimization algorithm, selecting the optimal algorithm, and discussing the influence of its sensitive variables and different weight assignments on the objective function under the algorithm. Through the above analysis, it serves as a reference for the design and comprehensive performance optimization of high-speed catamaran unmanned craft.

\section{OPTIMIZATION ALGORITHM}

\section{A. Genetic algorithm optimization \\ a. Concept and principle of genetic algorithm}

The Genetic Algorithm is a random search method derived from the evolutionary law of the biological world (the survival of the fittest, the genetic mechanism of the survival of the fittest). It starts from the initial population and repeats the operation of selection, crossover and mutation, so that the population develops along the established target from generation to generation.

The basic principle of genetic algorithms is based on Darwin's theory of evolution. Evolution believes that each species will adapt to the environment more and more in development, and individuals with high environmental adaptability will be preserved, that is the survival of the fittest. It is essentially a series of operations on chromosome patterns, which by selecting operators to inherit the good patterns in the current population into the next generation population, using crossover operators for pattern recombination, and using mutation operators for pattern mutation. Through these genetic operations, the pattern gradually evolves in a better direction, and finally we get the optimal solution to the problem ${ }^{[9]}$.

b. Basic operation of the genetic algorithm

1) Individual coding

Due to the robustness of the genetic a-lgorithm, it is not critical to the coding

requirements. Most problems can be expressed in the form of chromosomes in which

the genes are arranged in one dimension,

the most common being the binary encodin-g based on the $\{0$ , 1) symbol set.

2) Population size setting

Consider both speed and individual diversity. Quantitative research shows that when the genetic individual adopts binary coding, the coding length is $\mathrm{L}$, then the population size $n$ is satisfied with $n \geq 2^{L / 2}$. Consider increasing the size of the population as much as possible within the scope of computing power to ensure individual diversity and avoid falling into local optimum. 


\section{3) Generation of initial population individuals}

There are two ways, one is completely random, and the computer software calls a random function. The other is to use prior knowledge to transform a priori knowledge into a set of requirements that must be met, and then randomly select individuals in the solution that satisfies the requirements.

4) Determination of evolutionary algebra

In general, the more evolutionary algebras, the better individual performance. There are two ways to determine evolutionary algebra: one is to develop an evolutionary algebra according to the size of the solution space, and if the optimization result is not ideal, increase the number of iterations. The other is not to specify explicit algebra, but to determine whether to end evolution based on the performance of the optimal solution of evolution. For example, if the fitness value is required to be reached, or if the optimal individual fitness change for successive $\mathrm{n}$ generations is less than a specified threshold e, the cyclic evolution is stopped. But be sure to choose the appropriate threshold ${ }^{[12]}$.

5) Determination of the probability of crossover (the ratio of the number of descendants produced in each generation to the number of individuals in the population)

The general crossover probability is set to $0.4-0.8$.

6) Determination of the probability of variation (the percentage of the number of variant genes in the population in the total number of genes)

The value of the mutation probability is small, usually $0.001-0.1$

\section{c. Characteristics of genetic algorithm}

1) The genetic algorithm simultaneously processes multiple individuals in the group, which evaluates multiple solutions in the search space, reduces the risk of falling into the local optimal solution, and the algorithm itself is easy to implement parallelism.

2) The genetic algorithm basically does not use the knowledge of the search space or other auxiliary information, but only evaluates the individual with the fitness function value, and performs genetic operations on this basis. The fitness function is not only not subject to continuous and differentiable constraints, but its domain can be arbitrarily $\operatorname{set}^{[11]}$.

3) The genetic algorithm does not use deterministic rules, but uses the probability transition rules to guide its search direction.

4) Genetic algorithms are self-organizing, adaptive and self-learning. When the genetic algorithm uses the information obtained by the evolution process to organize the search by itself, the individuals with large fitness have higher survival probability and obtain a more adaptive genetic structure.

\section{B Particle Swarm Optimization}

a. Concept and principle of particle swarm optimization

Particle Swarm Optimization is an emerging heuristic global search algorithm whose basic idea is to simulate migration and clustering behavior during the foraging of birds. Similar to other evolutionary algorithms, which is based on the concepts of "population" and "evolution", searching for the optimal solution of complex space ${ }^{[1]}$ through collaboration and competition among individuals.

The principle is to initialize a group of random particles at the beginning, and then find the optimal solution through iteration. When searching in the space of solution, each particle will get two extreme values, one is the optimal solution found by itself, which called the individual extreme value, and the other is the optimal solution currently found by the whole population, namely the global pole. value. So in each iteration, the particles can finally get the position of the optimal particle by tracing the position of these two extremes and constantly updating their position ${ }^{[6]}$.

b. Basic operations of the particle swarm algorithm

1) Randomly initialize the particles in the group, including the random position and velocity, within a certain range according to the constraints.

2) Calculate the fitness value of each particle.

3) Compare the fitness value of each particle with the fitness value of the best position in its history, and store the individual extremum information.

4) Compare the historical optimal fitness value of each particle with the historical optimal fitness value within the group or neighborhood, and take the better value as the current global extreme value.

5) Update the velocity and position of the particle according to the particle position and velocity variation formula.

6) If the termination condition is not reached, go back to step 2.

Its flow chart is shown in Figure 2.1.

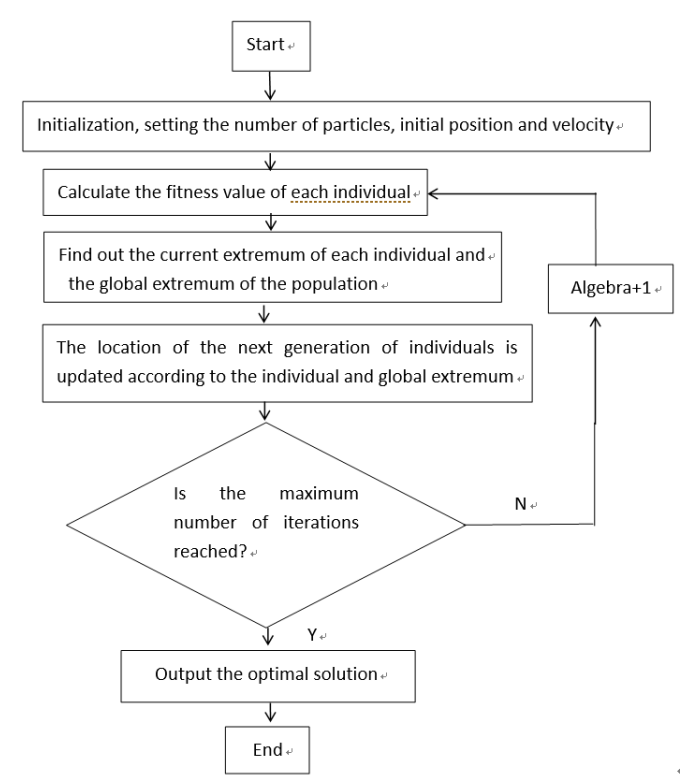

Figure 2.1 Particle Swarm Algorithm Flowchart

\section{c. Characteristics of Particle Swarm Algorithm}

The particle swarm optimization algorithm is characterized in that the particles in the population fly to the best position of their own history and the best position of the group history, so it is easy to fall into local extremum and premature convergence. In addition, it depends on the setting of algorithm parameters ${ }^{[10]}$. The parameters mainly have maximum speed, two acceleration constants and inertia constants. The setting of parameter values greatly affects the search ability of the algorithm. Since the motion trajectory generated by the particle position update formula is uncontrollable, this causes the particles to pulsate in the solution space, so we must use the maximum speed to limit the speed. Increasing the maximum speed is good for global search, and reducing the maximum speed is beneficial for local development. However, if the maximum speed is too high, the particle motion may exceed the position of the 
optimal solution, which makes the algorithm difficult to converge. On the contrary, the algorithm with too much maximum speed may fall into local extremum. The choice of maximum speed is usually given empirically and is typically set to $10-20 \%$ of the problem space. At present, particle swarm optimization has proven to be a simple and effective algorithm that has developed rapidly in recent years.

\section{MATHEMATICAL MODEL OF COMPREHENSIVE OPTIMIZATION CALCULATION OF NAVIGATION PERFORMANCE}

In this paper, the rapidity, maneuverability, and stability were used to optimize the objective function and construct its mathematical model. See below for a detailed description.

A. Design variables

Considering the rapidity, maneuverability and anti-overturning nature of unmanned crafts, this paper selects 16 design variables, namely: draft $\mathrm{T}$, propeller diameter $\mathrm{Dp}$, disk ratio Aeo, pitch ratio, speed N, Speed V, center of gravity $P_{D P}$ and depth ratio $\delta_{Z D}$, draft depth ratio TD, rudder angle $R a d$, sheet spacing $C_{0}$, total design waterline width $B_{0}$, ratio of length of the top superstructure to the length of the ground floor $\delta_{L 1}$, height of the top superstructure $\mathrm{H} 1$, length of the superstructure and length of the superstructure $\delta_{L 2}$, height of the superstructure $\mathrm{H} 2$, the ratio of the superstructure width to the ship width $\delta_{B a}$. Combine these 16 design variables in a single

vector:

$$
\begin{array}{r}
\left\{X_{S P}=T_{v} D_{p}, A_{e 0}, P_{D P}, N, V_{v} \delta_{Z D}, T D,\right. \\
\left.R a d_{s} C_{0,} B_{0,} \delta_{L 1,} H_{1,} \delta_{L 2}, H_{2,}, \delta_{D a}\right\}
\end{array}
$$

B. Objective function

The naval coefficient is a comprehensive evaluation factor that can be used to measure the ship's rapid performance. It can also measure the strength of the ship's line type and propeller's open water performance, and is more practical for ship design work $^{[8]}$. Fastness criterion $C_{\mathrm{e}}$ (similar to the naval coefficient) as a rapid optimization objective function: $f_{1}(x)=\frac{V^{3} \Delta^{2 / 3}}{p_{E}}=\frac{V^{3} \Delta^{2 / 3}\left(\eta_{0} \eta_{H} \eta_{R} \eta_{S}\right)}{R_{t} V * 0.5144 * 1.35962 * 10^{-3}}$

Where: Rt--- total resistance (unit: N); V--- design speed (unit: kn); $\Delta$--- displacement (unit: t); $\eta_{0}$--- propeller open water efficiency; $\eta_{H}$--- hull efficiency; $\eta_{R}$ - relative rotational efficiency; $\eta_{s}$--- shafting efficiency.

Ship maneuverability means that the ship can maintain or change the position, speed and heading performance according to the driver's intention, and is closely related to ship navigation safety and economy ${ }^{[7]}$.Considering the four aspects of ship maneuverability, this paper selected navigation stability, rotation and heading as indicators to measure the maneuverability of unmanned boats.

Selecting positive floating initial stability high GM and the inverse of the high stability after the overturning $G M_{1}^{\prime}$ to construct the anti-overturning optimization objective function. The specific expression is as follows:

$$
f_{3}(x)=G M^{\prime \gamma_{1}} * \overline{G M}_{1}{ }^{\gamma_{2}}
$$

In the formula: $G M^{\prime}$ and $\overline{G M_{1}}$ are the positive floating stability high GM and the opposite of high stability after the overturning ${\overline{G M_{1}}}^{\prime}$, which are corrected and compressed to the same order of magnitude. $\gamma_{1}, \gamma_{2}$ are the weights of positive float stability and self-righting performance, which

need to be met $\gamma_{1}, \gamma_{2}>0$ and $\gamma_{1}^{*} \gamma_{2}=1$

\section{Constraints}

There are two main types of constraints, namely equality constraints and inequality constraints.

Equality constraint:

1) The thrust constraint is satisfied, and the effective thrust of the propeller is equal to the total resistance of the hull. The expression is as follows:

$$
N_{p} K_{T} \rho N^{2} D_{P}^{4}(1-t)=R_{t}
$$

2) The torque constraint is satisfied. The torque supplied by the main engine to the propeller needs to be equal to the hydrodynamic torque that the propeller is subjected to. The expression is as follows:

$$
\frac{\eta_{R} \eta_{s} P_{s}}{2 \pi N}=K_{Q} \rho N^{2} D_{p}^{5}
$$

Inequality constraints:

1) The upper and lower limits of the design variables must be met ;

2)Propeller bubble constraint :

$$
\begin{aligned}
& A(x)=(1.3+0.3 Z) T_{e} /\left(\left(P_{0}-P_{V}\right) D_{P}^{2}\right)+ \\
& K-\left(A_{E} / A_{0}\right) \leq 0
\end{aligned}
$$

3) In order to meet the stability specifications of the ship, the initial stability of the initial float is greater than 0.3 .

\section{$G M>0.3 \quad$ (6)}

4) The total height of the superstructure should be greater than the draft.

\section{OPTIMIZATION CALCULATION AND RESULT ANALYSIS}

\section{A. Algorithm comparison and optimization result analysis}

In this paper, a high-speed catamaran unmanned craft with a displacement of 54t was used as a calculation and comparative analysis example. The genetic algorithm and the particle swarm algorithm were used to calculate and compare the objective functions, as shown in Table 1. According to the data in Table 1, it can be seen that the total objective function and the stability objective function obtained by the particle swarm optimization algorithm were higher than those of the genetic algorithm, and the fast objective function and the maneuvering objective function obtained by the two 
algorithms were basically the same. Therefore, for the high-speed catamaran unmanned craft in this experiment, the particle swarm optimization algorithm was superior to the genetic algorithm. Therefore, the particle swarm optimization algorithm was chosen to calculate the latter sensitive variables and the objective functions under different weights.

Table 1 Calculation results of each objective function

\begin{tabular}{|c|c|c|}
\hline Function value & Genetic algorithm & $\begin{array}{c}\text { Particle swarm } \\
\text { optimization }\end{array}$ \\
\hline $\begin{array}{c}\text { Total objective } \\
\text { function value }\end{array}$ & 88.39 & 96.29 \\
\hline $\begin{array}{c}\text { Rapid objective } \\
\text { function }\end{array}$ & 7.13 & 7.16 \\
\hline $\begin{array}{c}\text { Manipulative } \\
\text { objective function }\end{array}$ & 1.02 & 1.02 \\
\hline $\begin{array}{c}\text { Stability objective } \\
\text { function }\end{array}$ & 2.91 & 3.38 \\
\hline
\end{tabular}

B. Optimization calculation and result analysis of sensitive variables

Using the particle swarm algorithm, the five design variables were calculated (variables are propeller diameter, pitch ratio, speed, speed, and body spacing). The results were shown in figures $1,2,3,4$, and 5 , respectively. It can be seen from the figure that the change of the total objective function was the most obvious as the diameter of the propeller changes, so among these five variables, the diameter of the propeller was the most sensitive. As the pitch ratio changes, the change in the total objective function changes little, so in the above variables, the pitch ratio is the least sensitive variable. Therefore, we must pay attention to the setting of the propeller diameter when designing.

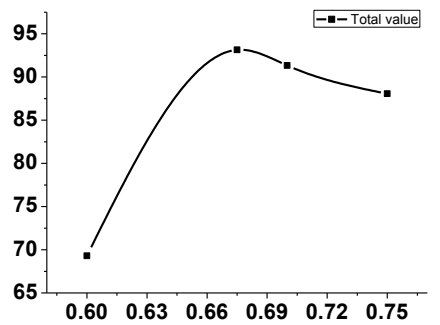

Figure 1 The total objective function value of the propeller diameter between 0.6 and 0.75

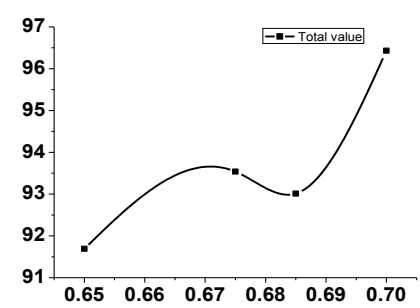

Figure 2 The total objective function value of the pitch ratio of $0.65-0.7$

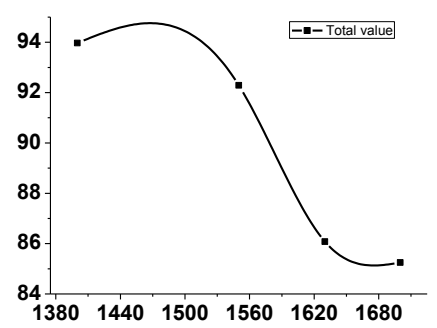

Figure 3 Total objective function of speed from 1400 to 1700

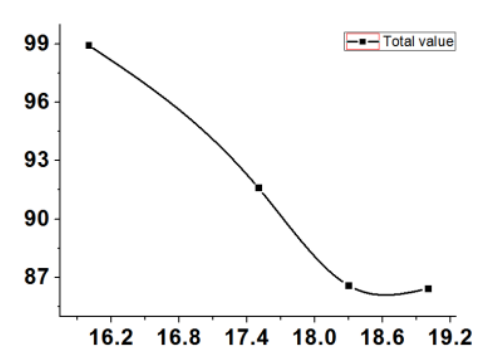

Figure 4 Total objective function of speed at 16-19

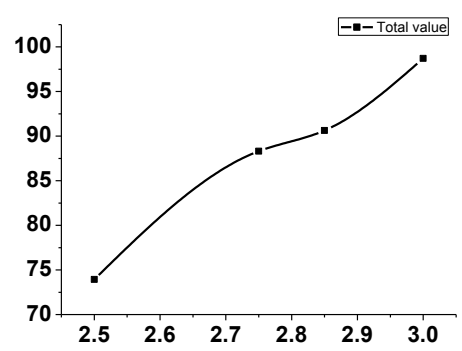

Figure 5 The total objective function of the slice spacing at $2.5-3$

\section{Optimization calculation and result analysis of different weights}

Discuss the objective functions under different weights, as shown in Table 7. As can be seen from Table 7, the change in weight has a great influence on the overall objective function. When selecting the weight, the influence of each part on the total objective function according to the actual navigation situation should not be based on the maximum total objective function as the measure of the best weight. Secondly, the equality constraint and the inequality constraint should be considered. Satisfy the situation. The inequality constraints of the above five sets of data are satisfied, and the fifth set of weights is best when the equality constraint is satisfied. Therefore, the optimized design of weights is important for the optimization analysis of navigation performance.

Table 7 Optimized calculated total objective function values under different weights

\begin{tabular}{|c|c|c|c|c|c|}
\hline $\begin{array}{c}\text { Rapid } \\
\text { weight }\end{array}$ & $\begin{array}{c}\text { Manipul } \\
\text { ative } \\
\text { weight }\end{array}$ & $\begin{array}{c}\text { Stability } \\
\text { weight }\end{array}$ & $\begin{array}{c}\text { Total } \\
\text { objective } \\
\text { function } \\
\text { value }\end{array}$ & $\begin{array}{c}\text { Force } \\
\text { meet / } \\
\%\end{array}$ & $\begin{array}{c}\text { Torque } \\
\text { satisfaction } \\
1 \%\end{array}$ \\
\hline 2 & 1 & 0.5 & 96.99 & 99.393 & 99.39 \\
\hline 1.5 & $7 / 4$ & $8 / 21$ & 31.16 & 99.083 & 99.075 \\
\hline $5 / 3$ & $3 / 5$ & 1 & 92.29 & 99.903 & 99.903 \\
\hline $8 / 5$ & $9 / 7$ & $35 / 72$ & 42.56 & 99.18 & 99.173 \\
\hline $9 / 5$ & $4 / 5$ & $25 / 36$ & 80.73 & 99.921 & 99.921 \\
\hline
\end{tabular}

\section{CONCLUSION}

This paper briefly introduced the related knowledge of genetic algorithm and particle swarm optimization algorithm and selected its design variables according to relevant data. It established a mathematical model with fast, maneuverability and anti-overturning as the objective function and its constraints. Genetic algorithm and particle swarm optimization algorithm are used to optimize the calculation, and the relevant data were obtained. The results show that the particle swarm optimization algorithm was better. Five variables were selected from the design variables, namely propeller diameter, pitch ratio, speed, speed, and body 
spacing. The objective function under different parameters was calculated. The results show that the diameter of the propeller was the most sensitive. Finally, the objective function under different weights was calculated. The results show that when the fastness weight was $9 / 5$, the maneuverability was $4 / 5$, and the stability was $25 / 36$, the result was the best, and the necessity of the weight coefficient was also explained. It provides a reference for the design and optimization of high-speed catamaran unmanned crafts.

\section{REFERENCE}

[1]Zheng Liexin. Modeling and motion control system control of surface unmanned boats [D]. South China University of Technology, 2018.

[2]Zhang Weiyi, Tang Wenyong, Wei Gang. Structural optimization of large-scale small-surface catamaran based on Asa algorithm [J]. Ship Science and Technology, 2016.5

[3] Deng Fang, Deng Weibin. Calculation of resistance performance and design of ship type for catamaran[J]. Journal of Qingdao University of Science and Technology(Natural Science), 2015, 36(1): 72-76.

[4] Zhai Aiqin, Liu Xinwang. Multi-target ship type optimization of ship wave resistance based on mspso algorithm [C]. Hydrodynamics Symposium, 2017.8

[5] Zou Ze,Cao Zhiwei. Yang Songlin.Study on the pitching motion model of a high-speed monohull ship based on test[C]. ICMMCCE,2017.

[6] Xue Min, Xu Haicheng. Path planning of unmanned boats based on particle swarm optimization algorithm $[\mathrm{J}]$. China Science and Technology Information, 2018.

[7] Liu Bing. Research on longitudinal motion and control of high-speed catamaran [D]. Harbin Engineering University, 2012

[8] Yu Ning. Preliminary study on comprehensive optimization method for navigation performance of small high-speed surface unmanned watercraft [D]. Master of Science in Engineering, Jiangsu University of Science and Technology. 2012, 3.

[9] Wei Zifan, Jing Shengping, Yang Songlin. Comprehensive optimization analysis of performance of new surface unmanned watercraft based on improved genetic algorithm [J]. Journal of Jiangsu University of Science and Technology, 2017.2

[10] Liu Yi. Improvement and application of particle swarm optimization algorithm [D]. Xidian University, 2013.

[11] Yang Songlin, Chen Peng, Wen Yiyan, Cui Jian, Chen Shuling. One Optimization Method on Mechanics Performance Reliability of the High-speed Monohull Ship[C].OMAE 2012 ;

[12]Nakamura M, Izui K, Nishiwaki S, et al. A Multi-Objective Particle Swarm Optimization Incorporating Design Sensitivities[C]. Aiaa/issmo Multidisciplinary Analysis and Optimization Conference. 2013 